\title{
Lower semicontinuity of solution mappings for parametric fixed point problems with applications*
}

\author{
Yu $\operatorname{Han}^{a}$ and Nan-jing Huang ${ }^{b \dagger}$ \\ a. Department of Mathematics, Nanchang University, Nanchang, Jiangxi 330031, P.R. China \\ b. Department of Mathematics, Sichuan University, Chengdu, Sichuan 610064, P.R. China
}

\begin{abstract}
In this paper, we establish the lower semicontinuity of the solution mapping and of the approximate solution mapping for parametric fixed point problems under some suitable conditions. As applications, the lower semicontinuity result applies to the parametric vector quasi-equilibrium problem, and allows to prove the existence of solutions for generalized Stackelberg games.
\end{abstract}

Keywords: Parametric fixed point problem; solution mapping; lower semicontinuity; generalized Stackelberg equilibrium problem; parametric vector quasiequilibrium problem.

2010 Mathematics Subject Classification: 54H25; 90C31; 91B50.

\section{Introduction}

The semicontinuity of solution mappings of vector equilibrium problems has been investigated by several authors, see [1,4, 6, 9, 12, 15, 17] and the references therein. Recently, in order to show the semicontinuity of the solution mappings for the parametric (vector) quasi-equilibrium problems, all the solution mappings of the parametric fixed point problems are assumed to be lower semicontinuous in the literature [1-3]. We note that in the literature mentioned above, the authors have not given any conditions to guarantee the lower semicontinuity of the solution mappings of the parametric fixed point problems. On the other hand, it is difficult to obtain the explicit solutions for some real problems when the data concerned with the problems are perturbed by noise and so the mathematical models are usually solved by numerical methods for approximating the exact solutions. Therefore, one nature question is: can we provide conditions ensuring the lower semicontinuity of the (approximate) solution mappings?

The main purpose of this paper is to make a new attempt to establish the lower semicontinuity of the solution mapping and of the approximate solution mapping for parametric fixed point problems under suitable conditions. The rest of the paper is organized as follows. Section 2 presents some necessary notations and lemmas. In Section 3, we establish the lower semicontinuity of the solution mapping and of the approximate solution mapping for parametric fixed point problems. In Section 4, the lower semicontinuity result applies to the parametric vector quasi-equilibrium problem, and allows to prove the existence of solutions for generalized Stackelberg games.

\footnotetext{
${ }^{*}$ This work was supported by the National Natural Science Foundation of China $(11471230,11671282)$.

†Corresponding author, E-mail: nanjinghuang@hotmail.com; njhuang@scu.edu.cn
} 


\section{Preliminaries}

Throughout this paper, unless otherwise specified, let $\Lambda$ and $X$ be two normed vector spaces, $\mathbb{R}_{+}=$ $\{x \in \mathbb{R}: x \geq 0\}, \mathbb{R}_{+}^{0}=\{x \in \mathbb{R}: x>0\}$ and $\mathbb{N}=\{1,2, \cdots\}$. Let $A$ be a nonempty subset of $X$ and $T: A \times \Lambda \rightarrow 2^{A}$ be a set-valued mapping. For $\lambda \in \Lambda$, we consider the following parametric fixed point problem consisting of finding $x_{0} \in A$ such that

$$
\text { (PFPP) } \quad x_{0} \in T\left(x_{0}, \lambda\right)
$$

For $\lambda \in \Lambda$, let $S(\lambda)$ denote the set of all solutions of (PFPP), i.e.

$$
S(\lambda)=\{x \in A: x \in T(x, \lambda)\}
$$

For $(\lambda, \varepsilon) \in \Lambda \times \mathbb{R}_{+}$, let $E(\lambda, \varepsilon)$ denote the set of all $\varepsilon$-approximate solutions of (PFPP), i.e.

$$
E(\lambda, \varepsilon)=\{x \in A: d(x, T(x, \lambda)) \leq \varepsilon\},
$$

where $d(x, T(x, \lambda))=\inf _{y \in T(x, \lambda)} d(x, y)$ and $d(x, y)=\|x-y\|$.

Denote the boundary of $D$ by $\partial D$, the complement of $D$ by $D^{c}$, the closure of $D$ by $\operatorname{cl} D$ and the interior of $D$ by int $D$.

Definition 2.1. ([16]) A nonempty convex subset $D$ of $X$ is said to be rotund if the boundary of $D$ does not contain line segments, i.e., for any $x_{1}, x_{2} \in D$ with $x_{1} \neq x_{2},\left(x_{1}, x_{2}\right) \cap(\partial D)^{c} \neq \emptyset$, where $\left(x_{1}, x_{2}\right)=$ $\left\{\lambda x_{1}+(1-\lambda) x_{2}: \lambda \in(0,1)\right\}$.

Remark 2.1. Let $D$ be a nonempty convex subset of $X$. Then it is easy to see that $D$ is rotund if and only if for any $x_{1}, x_{2} \in D$ with $x_{1} \neq x_{2}$, there exists $\lambda_{0} \in(0,1)$ such that $\lambda_{0} x_{1}+\left(1-\lambda_{0}\right) x_{2} \in \operatorname{int} D$. Let $D=\left\{(x, y) \in \mathbb{R}^{2}: x^{2}+y^{2} \leq 1\right\}$. Then it is clear that $D$ is rotund.

Definition 2.2. Let $\Delta$ and $\Delta_{1}$ be two topological vector spaces. A set-valued mapping $\Phi: \Delta \rightarrow 2^{\Delta_{1}}$ is said to be

(i) upper semicontinuous (u.s.c.) at $u_{0} \in \Delta$ if, for any neighborhood $V$ of $\Phi\left(u_{0}\right)$, there exists a neighborhood $U\left(u_{0}\right)$ of $u_{0}$ such that for every $u \in U\left(u_{0}\right), \Phi(u) \subseteq V$.

(ii) lower semicontinuous (l.s.c.) at $u_{0} \in \Delta$ if, for any $x \in \Phi\left(u_{0}\right)$ and any neighborhood $V$ of $x$, there exists a neighborhood $U\left(u_{0}\right)$ of $u_{0}$ such that for every $u \in U\left(u_{0}\right), \Phi(u) \cap V \neq \emptyset$.

(iii) Hausdorff lower semicontinuous (H-l.s.c.) at $u_{0} \in T$ if, for any neighborhood $V$ of $0 \in T_{1}$, there exists a neighborhood $U\left(u_{0}\right)$ of $u_{0}$ such that for every $u \in U\left(u_{0}\right), G\left(u_{0}\right) \subseteq G(u)+V$.

(iv) convex if, the graph of $\Phi$, i.e., Graph $(\Phi):=\left\{(x, y) \in \Delta \times \Delta_{1}: y \in \Phi(x)\right\}$ is a convex set in $\Delta \times \Delta_{1}$.

(v) rotund if, $\operatorname{Graph}(\Phi)$ is convex and for any $\left(x_{1}, y_{1}\right),\left(x_{2}, y_{2}\right) \in \operatorname{Graph}(\Phi)$ with $x_{1} \neq x_{2}, y_{1} \neq y_{2}$, we have

$$
\left(\left(x_{1}, y_{1}\right),\left(x_{2}, y_{2}\right)\right) \cap(\partial \operatorname{Graph}(\Phi))^{c} \neq \emptyset,
$$

where $\left(\left(x_{1}, y_{1}\right),\left(x_{2}, y_{2}\right)\right)=\left\{\lambda\left(x_{1}, y_{1}\right)+(1-\lambda)\left(x_{2}, y_{2}\right): \lambda \in(0,1)\right\}$. 
We say that $\Phi$ is u.s.c. and l.s.c. on $\Delta$ if it is u.s.c. and l.s.c. at each point $u \in \Delta$, respectively. $\Phi$ is called to be continuous on $\Delta$ if it is both u.s.c. and l.s.c. on $\Delta$.

Remark 2.2. Obviously, if $\Phi: \Delta \rightarrow 2^{\Delta_{1}}$ is convex, then $\Phi(x)$ is a convex set for any $x \in \Delta$.

Lemma 2.1. ( [5]) A set-valued mapping $\Phi: \Delta \rightarrow 2^{\Delta_{1}}$ is l.s.c. at $u_{0} \in \Delta$ if and only if for any sequence $\left\{u_{n}\right\} \subseteq \Delta$ with $u_{n} \rightarrow u_{0}$ and for any $x_{0} \in \Phi\left(u_{0}\right)$, there exists $x_{n} \in \Phi\left(u_{n}\right)$ such that $x_{n} \rightarrow x_{0}$.

Lemma 2.2. ( [10]) Let $\Phi: \Delta \rightarrow 2^{\Delta_{1}}$ be a set-valued mapping. For any given $u_{0} \in \Delta$, if $\Phi\left(u_{0}\right)$ is compact, then $\Phi$ is u.s.c. at $u_{0} \in \Delta$ if and only if for any sequence $\left\{u_{n}\right\} \subseteq \Delta$ with $u_{n} \rightarrow u_{0}$ and for any $x_{n} \in \Phi\left(u_{n}\right)$, there exist $x_{0} \in \Phi\left(u_{0}\right)$ and a subsequence $\left\{x_{n_{k}}\right\}$ of $\left\{x_{n}\right\}$ such that $x_{n_{k}} \rightarrow x_{0}$.

Lemma 2.3. (Kakutani-Fan-Glicksberg Fixed Point Theorem [7,8]]). Let $K$ be a nonempty compact convex subset of a locally convex Hausdorff topological vector space $X$ and let $F: K \rightarrow 2^{K}$ be an u.s.c. set-valued mapping with nonempty compact convex values. Then there exists $x_{0} \in K$ such that $x_{0} \in F\left(x_{0}\right)$.

\section{The main results}

Lemma 3.1. Let $X$ be a reflexive Banach space, $B$ be the closed unit ball of $X$ and $A$ be a nonempty closed convex subset of $X$. For given $\delta>0$, if $a+\delta B \subseteq A+\delta B$, then $a \in A$.

Proof Suppose on the contrary that $a \notin A$. Since $A$ is closed, one has

$$
d(a, A)=\inf _{y \in A}\|a-y\|>0 .
$$

Noting that $X$ is a reflexive Banach space and $A$ is a nonempty closed convex subset of $X$, there exists $\beta \in A$ such that

$$
d(a, A)=\inf _{y \in A}\|a-y\|=\|a-\beta\|>0 .
$$

Let $\lambda=\frac{\|a-\beta\|}{\delta+\|a-\beta\|}$. We choose $h \in X$ such that $a=\lambda h+(1-\lambda) \beta$. Then $h=\frac{a}{\lambda}+\beta-\frac{\beta}{\lambda}$.

We claim that $\|h-y\| \geq\|h-\beta\|$ for any $y \in A$. It follows from (10) that

$$
\|a-y\| \geq\|a-\beta\|, \quad \forall y \in A .
$$

For any $y \in A$, since $y, \beta \in A$ and $A$ is convex, we have $\lambda y+(1-\lambda) \beta \in A$. By (2), we know that $\|a-(\lambda y+(1-\lambda) \beta)\| \geq\|a-\beta\|$ and so

$$
\|h-y\| \geq\|h-\beta\|, \quad \forall y \in A .
$$

On the other hand,

$$
\|h-a\|=\left\|\frac{a}{\lambda}+\beta-\frac{\beta}{\lambda}-a\right\|=\left(\frac{1}{\lambda}-1\right)\|a-\beta\|=\delta
$$

and so $h \in a+\delta B$. Noting that (3) and

$$
\|h-\beta\|=\left\|\frac{a}{\lambda}+\beta-\frac{\beta}{\lambda}-\beta\right\|=\frac{1}{\lambda}\|a-\beta\|=\delta+\|a-\beta\|>\delta,
$$

we have $h \notin A+\delta B$ and so $a+\delta B \not \subset A+\delta B$, which contradicts the assumption that $a+\delta B \subseteq A+\delta B$. This completes the proof. 
Theorem 3.1. Let $\lambda_{0} \in \Lambda$ and $A$ be a nonempty compact convex subset of a reflexive Banach space $X$. Assume that $T\left(\cdot, \lambda_{0}\right)$ is rotund and $T(\cdot, \cdot)$ is continuous on $A \times\left\{\lambda_{0}\right\}$ with nonempty closed convex values. Then $S(\cdot)$ is l.s.c. at $\lambda_{0}$.

Proof Suppose on the contrary that $S(\cdot)$ is not l.s.c. at $\lambda_{0}$. Then there exist a point $x_{0} \in S\left(\lambda_{0}\right)$, a neighborhood $W_{0}$ of $0 \in X$ and a sequence $\left\{\lambda_{n}\right\}$ with $\lambda_{n} \rightarrow \lambda_{0}$ such that

$$
\left(x_{0}+W_{0}\right) \cap S\left(\lambda_{n}\right)=\emptyset, \quad \forall n \in \mathbb{N} .
$$

There are two cases to be considered.

Case 1. $S\left(\lambda_{0}\right)$ is a singleton. For $x_{n} \in S\left(\lambda_{n}\right)$, one has

$$
x_{n} \in T\left(x_{n}, \lambda_{n}\right), \quad \forall n \in \mathbb{N} .
$$

Since $x_{n} \in A$ and $A$ is compact, without loss of generality, we can assume that $x_{n} \rightarrow \bar{x} \in A$. Noting that $T(\cdot, \cdot)$ is u.s.c. at $\left(\bar{x}, \lambda_{0}\right)$, it follows from Lemma 2.2 and (5) that there exist a point $x^{\prime} \in T\left(\bar{x}, \lambda_{0}\right)$ and a subsequence $\left\{x_{n_{k}}\right\}$ of $\left\{x_{n}\right\}$ such that $x_{n_{k}} \rightarrow x^{\prime}$. By $x_{n} \rightarrow \bar{x}$, we know that $\bar{x}=x^{\prime}$ and so $\bar{x}=x^{\prime} \in T\left(\bar{x}, \lambda_{0}\right)$. This means that $\bar{x} \in S\left(\lambda_{0}\right)$. Noting that $S\left(\lambda_{0}\right)$ is a singleton, we have $\bar{x}=x_{0}$ and so $x_{n} \rightarrow \bar{x}=x_{0}$. Thus, $x_{n} \in x_{0}+W_{0}$ for $n$ large enough. This together with $x_{n} \in S\left(\lambda_{n}\right)$ implies that $\left(x_{0}+W_{0}\right) \cap S\left(\lambda_{n}\right) \neq \emptyset$ for $n$ large enough, which contradicts (4).

Case 2. $S\left(\lambda_{0}\right)$ is not a singleton. Then there exists $x^{*} \in S\left(\lambda_{0}\right)$ such that $x^{*} \neq x_{0}$. Since $x^{*}, x_{0} \in S\left(\lambda_{0}\right)$, we know that $x^{*} \in T\left(x^{*}, \lambda_{0}\right)$ and $x_{0} \in T\left(x_{0}, \lambda_{0}\right)$. Thus, $\left(x^{*}, x^{*}\right),\left(x_{0}, x_{0}\right) \in \operatorname{Graph}\left(T\left(\cdot, \lambda_{0}\right)\right)$. Let

$$
x(t)=t x^{*}+(1-t) x_{0}, \quad \forall t \in[0,1] .
$$

Then it is clear that $x(t) \in A$. Since $\operatorname{Graph}\left(T\left(\cdot, \lambda_{0}\right)\right)$ is rotund, we can find $t_{0} \in(0,1)$ such that

$$
x\left(t_{0}\right) \in x_{0}+W_{0}
$$

and

$$
\left(x\left(t_{0}\right), x\left(t_{0}\right)\right) \in \operatorname{int}\left(\operatorname{Graph}\left(T\left(\cdot, \lambda_{0}\right)\right)\right) .
$$

It follows from (7) that there exists a constant $\delta>0$ such that

$$
\left(x\left(t_{0}\right), x\left(t_{0}\right)\right)+\delta B \times \delta B \in \operatorname{Graph}\left(T\left(\cdot, \lambda_{0}\right)\right),
$$

where $B$ is the closed unit ball in $X$. This shows that

$$
x\left(t_{0}\right)+\delta B \subseteq T\left(x\left(t_{0}\right), \lambda_{0}\right) .
$$

Since $T\left(x\left(t_{0}\right), \cdot\right)$ is l.s.c. at $\lambda_{0}$ and $T\left(x\left(t_{0}\right), \lambda_{0}\right)$ is compact, we can see that $T\left(x\left(t_{0}\right), \cdot\right)$ is H-l.s.c. at $\lambda_{0}$. Thus, for $\delta B$, there exists $n_{0} \in \mathbb{N}$ large enough such that $T\left(x\left(t_{0}\right), \lambda_{0}\right) \subseteq T\left(x\left(t_{0}\right), \lambda_{n_{0}}\right)+\delta B$ and so (8) yields that

$$
x\left(t_{0}\right)+\delta B \subseteq T\left(x\left(t_{0}\right), \lambda_{n_{0}}\right)+\delta B .
$$

By the convexity and closedness of $T\left(x\left(t_{0}\right), \lambda_{n_{0}}\right)$, from (9) and Lemma 3.1, we know that $x\left(t_{0}\right) \in T\left(x\left(t_{0}\right), \lambda_{n_{0}}\right)$ and so $x\left(t_{0}\right) \in S\left(\lambda_{n_{0}}\right)$. This together with (6) implies that $x\left(t_{0}\right) \in\left(x_{0}+W_{0}\right) \cap S\left(\lambda_{n_{0}}\right)$, which contradicts (4). This completes the proof. 
Remark 3.1. In [1]3], the authors assume that $S(\cdot)$ is l.s.c. on $\Lambda$, but they do not give any sufficient condition guaranteeing that $S(\cdot)$ is l.s.c. on $\Lambda$. In Theorem 3.1, we give a sufficient condition for the lower semicontinuity of $S(\cdot)$.

Theorem 3.2. Let $\left(\lambda_{0}, \varepsilon_{0}\right) \in \Lambda \times \mathbb{R}_{+}^{0}$ and $A$ be a nonempty compact convex subset of a normed vector space $X$. Assume that $T\left(\cdot, \lambda_{0}\right)$ is convex, $T\left(\cdot, \lambda_{0}\right)$ is u.s.c on $A$ with nonempty closed values and for any $x \in A$, $T(x, \cdot)$ is l.s.c. at $\lambda_{0}$. Then $E(\cdot, \cdot)$ is l.s.c. at $\left(\lambda_{0}, \varepsilon_{0}\right)$.

Proof Suppose on the contrary that $E(\cdot, \cdot)$ is not l.s.c. at $\left(\lambda_{0}, \varepsilon_{0}\right)$. Then there exist a point $x_{0} \in E\left(\lambda_{0}, \varepsilon_{0}\right)$, a neighborhood $W_{0}$ of $0 \in X$ and a sequence $\left\{\left(\lambda_{n}, \varepsilon_{n}\right)\right\}$ with $\left(\lambda_{n}, \varepsilon_{n}\right) \rightarrow\left(\lambda_{0}, \varepsilon_{0}\right)$ such that

$$
\left(x_{0}+W_{0}\right) \cap E\left(\lambda_{n}, \varepsilon_{n}\right)=\emptyset, \quad \forall n \in \mathbb{N} .
$$

Define a set-valued mapping $Q: \mathbb{R}_{+} \rightarrow 2^{A}$ by

$$
Q(\varepsilon)=E\left(\lambda_{0}, \varepsilon\right)=\left\{x \in A: d\left(x, T\left(x, \lambda_{0}\right)\right) \leq \varepsilon\right\}, \quad \varepsilon \in \mathbb{R}_{+} .
$$

We claim that $Q(\cdot)$ is l.s.c. on $\mathbb{R}_{+}^{0}$. Suppose on the contrary that there exists $\varepsilon_{0} \in R_{+}^{0}$ such that $Q(\cdot)$ is not l.s.c. at $\varepsilon_{0}$. Then there exist a point $\bar{x} \in Q\left(\varepsilon_{0}\right)$, a neighborhood $U_{0}$ of $0 \in X$ and a sequence $\left\{\varepsilon_{n}\right\}$ with $\varepsilon_{n} \rightarrow \varepsilon_{0}$ such that

$$
\left(\bar{x}+U_{0}\right) \cap Q\left(\varepsilon_{n}\right)=\emptyset, \quad \forall n \in \mathbb{N} .
$$

It is easy to see that, if $0 \leq \alpha \leq \beta$, then $Q(\alpha) \subseteq Q(\beta)$. Suppose that $\varepsilon_{0} \leq \varepsilon_{n}$. Then $\bar{x} \in Q\left(\varepsilon_{0}\right) \subseteq Q\left(\varepsilon_{n}\right)$, which contradicts (11). Thus, we know that $\varepsilon_{0}>\varepsilon_{n}$ for any $n \in \mathbb{N}$. It follows from Lemma 2.3 and the closedness of $T\left(x, \lambda_{0}\right)$ that

$$
Q(0)=\left\{x \in A: d\left(x, T\left(x, \lambda_{0}\right)\right)=0\right\}=\left\{x \in A: x \in T\left(x, \lambda_{0}\right)\right\} \neq \emptyset .
$$

We choose $x^{\prime} \in Q(0)$. It follows from $\varepsilon_{n} \rightarrow \varepsilon_{0}$ that there exists $\varepsilon_{n_{0}}$ such that

$$
\varepsilon^{\prime} \bar{x}+\left(1-\varepsilon^{\prime}\right) x^{\prime}=\bar{x}+\left(1-\varepsilon^{\prime}\right)\left(x^{\prime}-\bar{x}\right) \in \bar{x}+U_{0},
$$

where $\varepsilon^{\prime}=\frac{\varepsilon_{n_{0}}}{\varepsilon_{0}}$.

Now we claim that $\varepsilon^{\prime} \bar{x}+\left(1-\varepsilon^{\prime}\right) x^{\prime} \in Q\left(\varepsilon_{n_{0}}\right)$. In fact, since $x^{\prime} \in Q(0)$, we have $x^{\prime} \in T\left(x^{\prime}, \lambda_{0}\right)$ and so $\left(x^{\prime}, x^{\prime}\right) \in \operatorname{Graph}\left(T\left(\cdot, \lambda_{0}\right)\right)$. It follows from $\bar{x} \in Q\left(\varepsilon_{0}\right)$ that $d\left(\bar{x}, T\left(\bar{x}, \lambda_{0}\right)\right) \leq \varepsilon_{0}$. Since $T\left(\bar{x}, \lambda_{0}\right)$ is compact, there exists $\bar{y} \in T\left(\bar{x}, \lambda_{0}\right)$ such that

$$
d(\bar{x}, \bar{y})=d\left(\bar{x}, T\left(\bar{x}, \lambda_{0}\right)\right) \leq \varepsilon_{0} .
$$

Thus, $\bar{y} \in T\left(\bar{x}, \lambda_{0}\right)$ shows that $(\bar{x}, \bar{y}) \in \operatorname{Graph}\left(T\left(\cdot, \lambda_{0}\right)\right)$. Since $\operatorname{Graph}\left(T\left(\cdot, \lambda_{0}\right)\right)$ is convex, one has

$$
\left(\varepsilon^{\prime} \bar{x}+\left(1-\varepsilon^{\prime}\right) x^{\prime}, \varepsilon^{\prime} \bar{y}+\left(1-\varepsilon^{\prime}\right) x^{\prime}\right) \in \operatorname{Graph}\left(T\left(\cdot, \lambda_{0}\right)\right)
$$

and so

$$
\varepsilon^{\prime} \bar{y}+\left(1-\varepsilon^{\prime}\right) x^{\prime} \in T\left(\varepsilon^{\prime} \bar{x}+\left(1-\varepsilon^{\prime}\right) x^{\prime}, \lambda_{0}\right) .
$$

Thus, it follows from (13) that

$$
\begin{aligned}
& d\left(\varepsilon^{\prime} \bar{x}+\left(1-\varepsilon^{\prime}\right) x^{\prime}, T\left(\varepsilon^{\prime} \bar{x}+\left(1-\varepsilon^{\prime}\right) x^{\prime}, \lambda_{0}\right)\right) \\
\leq & d\left(\varepsilon^{\prime} \bar{x}+\left(1-\varepsilon^{\prime}\right) x^{\prime}, \varepsilon^{\prime} \bar{y}+\left(1-\varepsilon^{\prime}\right) x^{\prime}\right) \\
= & \varepsilon^{\prime} d(\bar{x}, \bar{y}) \leq \varepsilon_{n_{0}} .
\end{aligned}
$$


This means that $\varepsilon^{\prime} x+\left(1-\varepsilon^{\prime}\right) x^{\prime} \in Q\left(\varepsilon_{n_{0}}\right)$. It follows from (12) that

$$
\varepsilon^{\prime} \bar{x}+\left(1-\varepsilon^{\prime}\right) x^{\prime} \in\left(\bar{x}+U_{0}\right) \cap Q\left(\varepsilon_{n_{0}}\right),
$$

which contradicts (11). Therefore, $Q(\cdot)$ is l.s.c. on $\mathbb{R}_{+}^{0}$.

For the above $x_{0} \in E\left(\lambda_{0}, \varepsilon_{0}\right)=Q\left(\varepsilon_{0}\right)$ and $W_{0}$, there exists a neighborhood $U\left(\varepsilon_{0}\right)$ of $\varepsilon_{0}$ such that

$$
\left(x_{0}+W_{0}\right) \cap Q(\varepsilon) \neq \emptyset, \quad \forall \varepsilon \in U\left(\varepsilon_{0}\right) .
$$

Choose $\varepsilon^{*} \in U\left(\varepsilon_{0}\right)$ with $0<\varepsilon^{*}<\varepsilon_{0}$. Then

$$
\left(x_{0}+W_{0}\right) \cap E\left(u_{0}, \varepsilon^{*}\right)=\left(x_{0}+W_{0}\right) \cap Q\left(\varepsilon^{*}\right) \neq \emptyset
$$

and so there exists $x^{*} \in x_{0}+W_{0}$ such that

$$
d\left(x^{*}, T\left(x^{*}, \lambda_{0}\right)\right) \leq \varepsilon^{*} .
$$

It follows from $x^{*} \in x_{0}+W_{0}$ and (10) that $x^{*} \notin E\left(\lambda_{n}, \varepsilon_{n}\right)$ and so

$$
d\left(x^{*}, T\left(x^{*}, \lambda_{n}\right)\right)>\varepsilon_{n} .
$$

Let $\delta=\frac{\varepsilon_{0}-\varepsilon^{*}}{2}>0$. By (14), we know that there exists $y^{*} \in T\left(x^{*}, \lambda_{0}\right)$ such that

$$
d\left(x^{*}, y^{*}\right)<\varepsilon^{*}+\delta .
$$

Since $T\left(x^{*}, \cdot\right)$ is l.s.c. at $\lambda_{0}$, by Lemma 2.1] there exists $y_{n} \in T\left(x^{*}, \lambda_{n}\right)$ such that $y_{n} \rightarrow y^{*}$ and so $d\left(x^{*}, y_{n}\right) \rightarrow d\left(x^{*}, y^{*}\right)$. It follows from (16) that

$$
d\left(x^{*}, y_{n}\right)<\varepsilon^{*}+\delta
$$

for $n$ large enough. On the other hand, from (15) and $\varepsilon_{n} \rightarrow \varepsilon_{0}$, we have

$$
d\left(x^{*}, y_{n}\right) \geq d\left(x^{*}, T\left(x^{*}, \lambda_{n}\right)\right)>\varepsilon_{n}>\varepsilon_{0}-\delta=\varepsilon^{*}+\delta
$$

for $n$ large enough, which contradicts (17). This completes the proof.

Next, we give an example to illustrate Theorems 3.1 and 3.2 .

Example 3.1. Let $A=[0,2]$ and $\Lambda=\mathbb{R}$. Let $T: A \times \Lambda \rightarrow 2^{A}$ be defined as follows:

$$
T(x, \lambda)=\left\{y \in \mathbb{R}:\left(\frac{1}{3} \cos \lambda+\frac{2}{3}\right)\left(1-\sqrt{x^{2}+2 x}\right) \leq y \leq\left(\frac{1}{3} \cos \lambda+\frac{2}{3}\right)\left(1+\sqrt{x^{2}+2 x}\right)\right\} .
$$

Let $\lambda_{0}=0$ and $\varepsilon_{0}>0$. Clearly,

$$
\operatorname{Graph}\left(T\left(\cdot, \lambda_{0}\right)\right)=\left\{(x, y) \in \mathbb{R}^{2}:(x-1)^{2}+(y-1)^{2} \leq 1\right\}
$$

is rotund. It is easy to check that all conditions of Theorems 3.1 and 3.2 are satisfied. Thus, Theorem 3.1 shows that $S(\cdot)$ is l.s.c. at $\lambda_{0}$ and Theorem 3.2 implies that $E(\cdot, \cdot)$ is l.s.c. at $\left(\lambda_{0}, \varepsilon_{0}\right)$. 


\section{Applications}

\subsection{Existence of solutions for a class of generalized Stackelberg equilibrium problems}

Following Nagy [18, assume that $f_{1}, f_{2}: \mathbb{R}^{N} \times \mathbb{R}^{N} \rightarrow \mathbb{R}$ are the payoff/loss functions for two players, and $K_{1}, K_{2} \subset \mathbb{R}^{N}$ are their strategy sets. It is well known that the framework of Stackelberg equilibrium problem can be modelled by the following bi-level mathematical programming problem:

$$
\begin{aligned}
& \min f_{1}(x, y) \\
& \text { s.t. } \quad y \in R_{S E}(x), x \in K_{1},
\end{aligned}
$$

where $R_{S E}(x)$ denotes the Stackelberg equilibrium response set given by

$$
R_{S E}(x)=\left\{y \in K_{2}: f_{2}(x, v)-f_{2}(x, y) \geq 0, \forall v \in K_{2}\right\} .
$$

Applying the variational inequality theory and the fixed point theorem, Nagy [18 studied the existence and location of Stackelberg equilibrium problem under the assumptions that $f_{1}$ and $f_{2}$ are both smooth functions. Moreover, Han and Huang [13] showed the existence of solutions for the Stackelberg equilibrium problem without the smoothness by employing the lower semicontinuity of the set-valued mapping $R_{S E}(x)$.

Let $K_{1}, K_{2} \subset \mathbb{R}^{N}$ be two subsets and $f: \mathbb{R}^{N} \times \mathbb{R}^{N} \rightarrow \mathbb{R}$ be a function. Assume that $T: K_{2} \times K_{1} \rightarrow 2^{K_{2}}$ is a set-valued mapping. We consider the following generalized Stackelberg equilibrium problem:

$$
\begin{aligned}
& \min f(x, y) \\
& \text { s.t. } y \in R_{S E}^{\prime}(x), x \in K_{1},
\end{aligned}
$$

where $R_{S E}^{\prime}(x)$ is the Stackelberg equilibrium response set defined by

$$
R_{S E}^{\prime}(x)=\left\{y \in K_{2}: y \in T(y, x)\right\} .
$$

It is well known that, when $f_{2}$ is smooth or subdifferential, $y \in R_{S E}(x)$ if and only if $y$ is a fixed point of the single-valued or set-valued mapping. Therefore, the generalized Stackelberg equilibrium problem can be regarded as a generalization of the Stackelberg equilibrium problem considered by Nagy [18.

Now we are going to give an existence result concerned with the solutions of the generalized Stackelberg equilibrium problem. To this end, we assume that $K_{2}$ is a nonempty compact convex subset of $\mathbb{R}^{N}, T(\cdot, x)$ is rotund for any $x \in K_{1}$, and $T(\cdot, \cdot)$ is continuous on $K_{2} \times K_{1}$ with nonempty closed values. By Lemma 2.3, we can see that $R_{S E}^{\prime}(x)$ is nonempty for any $x \in K_{1}$. Since $T(\cdot, x)$ is convex, it is easy to see that $R_{S E}^{\prime}(x)$ is convex for any $x \in K_{1}$.

We claim that $R_{S E}^{\prime}(x)$ is closed for any $x \in K_{1}$. In fact, let $\left\{y_{n}\right\} \subseteq R_{S E}^{\prime}(x)$ with $y_{n} \rightarrow y_{0}$. Then $y_{0} \in K_{2}$ and $y_{n} \in T\left(y_{n}, x\right)$. Noting that $T(\cdot, x)$ is u.s.c. at $y_{0}$, it follows from Lemma 2.2 that there exist a point $y^{\prime} \in T\left(y_{0}, x\right)$ and a subsequence $\left\{y_{n_{k}}\right\}$ of $\left\{y_{n}\right\}$ such that $y_{n_{k}} \rightarrow y^{\prime}$. By $y_{n} \rightarrow y_{0}$, we have $y_{0}=y^{\prime}$ and so $y_{0}=y^{\prime} \in T\left(y_{0}, x\right)$. This means that $y_{0} \in R_{S E}^{\prime}(x)$ and so $R_{S E}^{\prime}(x)$ is closed.

Therefore, it follows from Theorem 3.1 that $R_{S E}^{\prime}: K_{1} \rightarrow 2^{K_{2}}$ is lower semi-continuous on $K_{1}$. By Michael's continuous selection theorem (see, for example, Theorem 16.1 of [11]), there exists a continuous selection $r(x) \in R_{S E}^{\prime}(x)$ for all $x \in K_{1}$. 
Moreover, we assume that $K_{1}$ is a nonempty compact subset of $\mathbb{R}^{N}$ and $f$ is continuous on $K_{1} \times K_{2}$. Then it is easy to see that $f(x, r(x))$ is continuous on $K_{1}$ and so there exists a point $x^{*} \in K_{1}$ such that

$$
f\left(x^{*}, r\left(x^{*}\right)\right)=\min _{x \in K_{1}} f(x, r(x)) .
$$

Let $y^{*}=r\left(x^{*}\right)$. Then we know that $\left(x^{*}, y^{*}\right)$ is a solution of the generalized Stackelberg equilibrium problem.

\subsection{Lower semicontinuity of the solution mapping for the parametric vector quasiequilibrium problems}

Let $\Lambda, \Omega$ and $Y$ be three normed vector spaces. Let $D$ be a nonempty subset of a reflexive Banach space $X$. Let $K: D \times \Lambda \rightarrow 2^{D}$ and $F: D \times D \times \Omega \rightarrow 2^{Y}$ be two set-valued mappings. Let $C \subseteq Y$ be closed with $\operatorname{int} C \neq \emptyset$. For $(u, \lambda) \in \Omega \times \Lambda$, we consider the following parametric vector quasiequilibrium problems:

(QEP) finding $x_{0} \in \operatorname{cl} K\left(x_{0}, \lambda\right)$ such that $\quad F\left(x_{0}, y, u\right) \cap(Y \backslash-\operatorname{int} C) \neq \emptyset, \quad \mathrm{y} \in K\left(x_{0}, \lambda\right)$;

(SQEP) finding $x_{0} \in \operatorname{cl} K\left(x_{0}, \lambda\right)$ such that $\quad F\left(x_{0}, y, u\right) \subseteq Y \backslash-\operatorname{int} C, \quad \mathrm{y} \in K\left(x_{0}, \lambda\right)$.

For $(u, \lambda) \in \Omega \times \Lambda$, let $M_{1}(u, \lambda)$ denote the set of all solutions of (QEP), i.e.

$$
M_{1}(u, \lambda)=\{x \in \operatorname{cl} K(x, \lambda): F(x, y, u) \cap(Y \backslash-\operatorname{int} C) \neq \emptyset, \forall y \in K(x, \lambda)\},
$$

and let $M_{2}(u, \lambda)$ denote the set of all solutions of (SQEP), i.e.

$$
M_{2}(u, \lambda)=\{x \in \operatorname{cl} K(x, \lambda): F(x, y, u) \subseteq Y \backslash-\operatorname{int} C, \forall y \in K(x, \lambda)\} .
$$

For $\lambda \in \Lambda$, let $H(\lambda):=\{x \in X: x \in \operatorname{cl} K(x, \lambda)\}$. We always assume that $M_{1}(u, \lambda) \neq \emptyset$ and $M_{2}(u, \lambda) \neq \emptyset$ for all $\lambda$ in a neighborhood of $\lambda_{0} \in \Lambda$ and for all $u$ in a neighborhood of $u_{0} \in \Omega$.

Form Theorem 2.1 of [1] and Theorem 3.1, we can get the following theorem.

Theorem 4.1. Let $\left(u_{0}, \lambda_{0}\right) \in \Omega \times \Lambda$ and $D$ be compact and convex. Assume that

(i) $K\left(\cdot, \lambda_{0}\right)$ is rotund and $K(\cdot, \cdot)$ is continuous on $D \times\left\{\lambda_{0}\right\}$ with nonempty closed convex values;

(ii) $F(\cdot, \cdot, \cdot)$ is l.s.c. on $D \times D \times\left\{u_{0}\right\}$;

(iii) for any $x \in M_{1}\left(u_{0}, \lambda_{0}\right)$ and any $y \in K\left(x, \lambda_{0}\right), F\left(x, y, u_{0}\right) \cap(Y \backslash-C) \neq \emptyset$.

Then $M_{1}(\cdot, \cdot)$ is l.s.c. at $\left(u_{0}, \lambda_{0}\right)$.

Definition 4.1. ([1]) Let $X$ be a Hausdorff topological space, $Y$ be a topological vector space and $C \subseteq Y$ with int $C \neq \emptyset$. A set-valued mapping $\Phi: X \rightarrow 2^{Y}$ is said to be have

(i) the $C$-inclusion property at $x_{0}$ if, for any $x_{\alpha} \rightarrow x_{0}$,

$$
\Phi\left(x_{0}\right) \cap(Y \backslash-\operatorname{int} C) \neq \emptyset \Rightarrow \exists \bar{\alpha}, \Phi\left(x_{\bar{\alpha}}\right) \cap(Y \backslash-\operatorname{int} C) \neq \emptyset .
$$

(ii) the strict $C$-inclusion property at $x_{0}$ if, for any $x_{\alpha} \rightarrow x_{0}$,

$$
\Phi\left(x_{0}\right) \subseteq Y \backslash-\operatorname{int} C \Rightarrow \exists \bar{\alpha}, \Phi\left(x_{\bar{\alpha}}\right) \subseteq Y \backslash-\operatorname{int} C .
$$


Form Theorem 2.2 of [1] and Theorem 3.1, we can get the following theorem.

Theorem 4.2. Let $\left(u_{0}, \lambda_{0}\right) \in \Omega \times \Lambda$ and $D$ be compact and convex. Assume that

(i) $K\left(\cdot, \lambda_{0}\right)$ is rotund and $K(\cdot, \cdot)$ is continuous on $D \times\left\{\lambda_{0}\right\}$ with nonempty closed convex values;

(ii) $F(\cdot, \cdot, \cdot)$ has the $C$-inclusion property on $D \times D \times\left\{u_{0}\right\}$.

Then $M_{1}(\cdot, \cdot)$ is l.s.c. at $\left(u_{0}, \lambda_{0}\right)$.

Form Theorem 2.3 of 1 and Theorem 3.1, we can get the following theorem.

Theorem 4.3. Let $\left(u_{0}, \lambda_{0}\right) \in \Omega \times \Lambda$ and $D$ be compact and convex. Assume that

(i) $K\left(\cdot, \lambda_{0}\right)$ is rotund and $K(\cdot, \cdot)$ is continuous on $D \times\left\{\lambda_{0}\right\}$ with nonempty closed convex values;

(ii) $F(\cdot, \cdot, \cdot)$ is u.s.c. on $D \times D \times\left\{u_{0}\right\}$;

(iii) for any $x \in M_{2}\left(u_{0}, \lambda_{0}\right)$ and any $y \in K\left(x, \lambda_{0}\right), F\left(x, y, u_{0}\right) \subseteq Y \backslash-C$.

Then $M_{2}(\cdot, \cdot)$ is l.s.c. at $\left(u_{0}, \lambda_{0}\right)$.

Form Theorem 2.4 of [1] and Theorem 3.1, we can get the following theorem.

Theorem 4.4. Let $\left(u_{0}, \lambda_{0}\right) \in \Omega \times \Lambda$ and $D$ be compact and convex. Assume that

(i) $K\left(\cdot, \lambda_{0}\right)$ is rotund and $K(\cdot, \cdot)$ is continuous on $D \times\left\{\lambda_{0}\right\}$ with nonempty closed convex values;

(ii) $F(\cdot, \cdot, \cdot)$ has the strict $C$-inclusion property on $D \times D \times\left\{u_{0}\right\}$.

Then $M_{2}(\cdot, \cdot)$ is l.s.c. at $\left(u_{0}, \lambda_{0}\right)$.

\section{Acknowledgements}

The authors are grateful to the editor and the referees for their valuable comments and suggestions.

\section{References}

[1] L.Q. Anh, P.Q. Khanh, Semicontinuity of the solution set of parametric multivalued vector quasiequilibrium problems, J. Math. Anal. Appl. 294 (2004) 699-711.

[2] L.Q. Anh, P.Q. Khanh, On the stability of the solution sets of general multivalued vector quasiequilibrium problems, J. Optim. Theory Appl. 135 (2007) 271-284.

[3] L.Q. Anh, P.Q. Khanh, Continuity of solution maps of parametric quasiequilibrium problems, J. Global Optim. 46 (2010) 247-259.

[4] L.Q. Anh, T.N. Tam, Hausdorff continuity of approximate solution maps to parametric primal and dual equilibrium problems, TOP 24 (2016) 242-258. 
[5] J.P. Aubin, I. Ekeland, Applied Nonlinear Analysis. Wiley, New York (1984)

[6] C.R. Chen, S.J. Li, K.L. Teo, Solution semicontinuity of parametric generalized vector equilibrium problems, J. Global Optim. 45 (2009) 309-318.

[7] K. Fan, Fixed point and minimax theorems in locally convex topological linear spaces, Proc. Natl. Acad. Sci. USA 38 (1952) 121-126.

[8] I. Glicksberg, A further generalization of Kakutani fixed point theorem with applications to Nash equilibrium points, Proc. Amer. Math. Soc. 3 (1952) 170-174.

[9] X.H. Gong, J.C. Yao, Lower semicontinuity of the set of efficient solutions for generalized systems, J. Optim. Theory Appl. 138 (2008) 197-205.

[10] A. Göpfert, H. Riahi, C. Tammer, C. Zălinescu, Variational Methods in Partially Ordered Spaces, Springer/Berlin Heidelberg/New York, (2003).

[11] L. Górniewicz, Topological Fixed Point Theorey of Multivalued Mappings, Kluwer Academic Publishers, Dordrecht/Boston/London, (1999).

[12] Y. Han, X.H. Gong, Lower semicontinuity of solution mapping to parametric generalized strong vector equilibrium problems, Appl. Math. Lett. 28 (2014) 38-41.

[13] Y. Han, N.J. Huang, Existence and stability of solutions for a class of generalized vector equilibrium problems, Positivity 20 (2016) 829-846.

[14] Y. Han, N.J. Huang, Some characterizations of the approximate solutions to generalized vector equilibrium problems, J. Ind. Manag. Optim. 12 (2016) 1135-1151.

[15] N.J. Huang, J. Li, H.B. Thompson, Stability for parametric implicit vector equilibrium problems, Math. Comput. Model. 43 (2006) 1267-1274.

[16] R.B. Holmes, Geometric Functional Analysis and Its Applications, Grad Texts in Mathematics 24. Springer, New York (1975).

[17] S.J. Li, H.M. Liu, Y. Zhang, Z.M. Fang, Continuity of the solution mappings to parametric generalized strong vector equilibrium problems, J. Global Optim. 55 (2013) 597-610.

[18] S. Nagy, Stackelberg equilibria via variational inequalities and projections, J. Global Optim. 57 (2013) 821-828. 